\title{
L'introduction du travail manuel dans les écoles primaires de garçons, 1880-1900
}

The Introduction of Craft into Primary Schools for Boys, 1880-1900

Zur Einführung des Werkunterrichts an Knabenvolksschulen zwischen 1880 und 1900

La introducción del trabajo manual en las escuelas primarias de niños

(1880-1900)

Renaud Enfert (d')

\section{OpenEdition}

Journals

Édition électronique

URL : https://journals.openedition.org/histoire-education/1353

DOI : 10.4000/histoire-education.1353

ISSN : 2102-5452

\section{Éditeur}

ENS Éditions

\section{Édition imprimée}

Date de publication : 1 janvier 2007

ISBN : 978-2-7342-1076-4

ISSN : 0221-6280

\section{Référence électronique}

Renaud Enfert (d'), «L'introduction du travail manuel dans les écoles primaires de garçons,

1880-1900 ", Histoire de l'éducation [En ligne], 113 | 2007, mis en ligne le 01 janvier 2012, consulté le 20 mai 2021. URL : http://journals.openedition.org/histoire-education/1353 ; DOI : https://doi.org/ 10.4000/histoire-education. 1353 


\section{L'INTRODUCTION DU TRAVAIL MANUEL dans les écoles primaires de garçons, 1880-1900}

par Renaud d'ENFERT

L'historiographie de l'éducation, enseignement technique inclus, offre une image assez paradoxale de l'enseignement du travail manuel dans les écoles primaires élémentaires de garçons des premières décennies de la Troisième République. Alors que l'iconographie mobilisée présente des écoliers s'activant à des travaux de menuiserie ou de ferronnerie (1), la plupart des synthèses historiques estiment que, faute d'ateliers en nombre suffisant, cet enseignement aurait été «négligé », « oublié », qu'il n'aurait existé qu' « en théorie », que ce serait un « échec » (2). En somme, à l'exception de quelques cas isolés mais exemplaires, la disposition de la loi du 28 mars 1882 , qui introduit pour la première fois les «travaux manuels » et l'« usage des outils des principaux métiers » dans l'enseignement primaire, n'aurait pas été appliquée.

Largement influencées par les études produites au début du $\mathrm{XX}^{\mathrm{e}}$ siècle (3), c'est-à-dire à un moment où l'enseignement technique commence à s'affirmer comme un ordre d'enseignement à part entière, de

(1) Collectif, Le Patrimoine de l'éducation nationale, Charenton-le-Pont, Flohic, 1999, p. 614; Yves Gaulupeau, La France à l'école, Paris, Gallimard, 1992, p. 100; Françoise Mayeur, Histoire générale de l'enseignement et de l'éducation en France. Tome 3: De la Révolution à l'École républicaine (1789-1930), Paris, Nouvelle Librairie de France, 1981, p. 551.

(2) Voir par exemple Béatrice Compagnon, Anne Thévenin, L'École et la société française, Bruxelles, Complexe, 1995, p. 39; Antoine Prost, Histoire de l'enseignement en France, 1800-1967, Paris, A. Colin, 1968, p. 341.

(3) Notamment Jean-Baptiste Paquier, L'Enseignement professionnel en France. Son histoire, ses différentes formes, ses résultats, Paris, A. Colin, 1908, pp. 107-124. Son point de vue est repris dans l'ouvrage de Jean-Pierre Guinot, Formation professionnelle et travailleurs qualifiés depuis 1789, Paris, Domat-Monchrestien, 1946, sur lequel s'appuient de nombreuses études. Pour une critique de ces dernières, voir JeanMichel Chapoulie, "Deux expériences de création d'établissements techniques au 
telles analyses proposent une conception passablement idéalisée de l'enseignement manuel à l'école, dont les exercices d'atelier ne seraient, en définitive, que les seules formes légitimes. Formuler l'hypothèse de l'échec, c'est, en effet, admettre une hiérarchie des activités manuelles scolaires, selon une échelle de valeur qui place au premier rang les modalités d'apprentissage des métiers auxquels sont a priori destinés les élèves du primaire. D'où l'idée, largement répandue, que toute autre espèce d'activité n'est pas du « vrai » travail manuel, a fortiori quand elle est effectuée en classe et non à l'atelier. D'où, également, la stigmatisation d'une institution scolaire, en l'occurrence l'école primaire, décidément incapable de prendre en compte la réalité des métiers et de transmettre des savoirs professionnels.

Bien que l'idée de l'échec de l'introduction du travail manuel à l'école semble faire l'unanimité, elle ne semble guère appuyée sur des faits précis. À notre connaissance, aucune enquête historique n'a été réalisée pour en quantifier l'ampleur. Le fiasco est-il total? Si l'on excepte le cas de Paris, souvent cité en exemple pour le nombre impressionnant de ses ateliers scolaires, on sait peu de chose, en réalité, sur l'implantation de tels ateliers dans le reste de la France. Selon Jean-Pierre Briand, vers 1900, « de nombreuses écoles primaires de garçons dans les grandes villes possèdent des ateliers » (1). Reste à savoir lesquelles, dans quelles proportions et quels élèves étaient concernés; reste à savoir également ce qu'il en est dans les villes de taille plus modeste. Par ailleurs, peu d'études ont tenté d'expliquer les raisons pour lesquelles la formule de l'atelier ne s'est pas généralisée. Est-ce le manque de moyens, l'absence de volonté politique? Si les créations d'ateliers dans les établissements d'enseignement « intermédiaire » peuvent sembler banales dans les années 1880, la législation de 1882 et la réglementation qui l'accompagne créent, au niveau de l'enseignement primaire élémentaire, une situation entièrement nouvelle qu'il convient d'analyser en détail afin d'en restituer la complexité.

$\mathrm{XIX}^{\mathrm{e}}$ siècle », Formation-Emploi, $\mathrm{n}^{\circ}$ spécial L'Enseignement technique et professionnel. Repères dans l'histoire (1830-1960), n 27-28, juillet-décembre 1989, pp. 15-39, et Philippe Savoie, "Société et école: réflexions autour de l'enseignement technique français (XIX'-XX ${ }^{\mathrm{e}}$ siècles) », Paedagogica Historica, Supplementary series, vol. IV, 1998, pp. 69-88.

(1) Jean-Pierre Briand, «L'apparition du "préapprentissage" dans les grandes villes au début du $\mathrm{XX}^{\mathrm{e}}$ siècle », Formation-Emploi, ${ }^{\circ}$ spécial L'Enseignement technique et professionnel..., op. cit., p. 43. 
Pour autant, la tâche ne serait qu'à moitié remplie. La discipline elle-même doit être interrogée. Curieusement, l'enseignement manuel n'a guère retenu l'attention des historiens de l'éducation, hormis quelques lignes dans les grandes synthèses. Peut-être leur faible intérêt à son égard provient-il du fait que, outre son statut de discipline mineure et sans grand enjeu idéologique a priori, le travail manuel apparaît inclassable, entre enseignement technique et enseignement général. On peut s'en étonner, alors que le travail manuel apparaît comme un constituant spécifique de la culture primaire et un élément essentiel de la différenciation entre les ordres primaire et secondaire, du moins dans les premières décennies de la Troisième République. L'histoire de l'enseignement technique ne s'est pas davantage emparée de la question. Bien souvent, en effet, ce domaine d'étude privilégie les approches institutionnelles plutôt que disciplinaires, et les logiques d'établissement plutôt que les enseignements dispensés. En revanche, d'autres champs de recherche, comme la sociologie ou la didactique - avec les travaux de Joël Lebeaume notamment -, se sont intéressés à l'introduction du travail manuel à l'école (1). Mais l'interprétation de celle-ci comme scolarisation de pratiques sociales revient à mettre en doute la légitimité de l'enseignement manuel tel qu'il se développe effectivement dans l'espace scolaire, c'est-à-dire avec des contenus et des finalités largement reformulés par l'école.

Écartant $a$ priori l'hypothèse de l'échec, cet article voudrait montrer que l'introduction du travail manuel à l'école primaire dans les années 1880-1900 ne se limite pas à une simple importation/adaptation de savoirs et de pratiques venus de l'extérieur, mais que d'autres formes de travail manuel, conçues par l'école et pour l'école, se mettent en place dans cette période, constituant le noyau dur de ce nouvel enseignement. Laissant volontairement de côté l'enseignement féminin, qui relève d'une tradition différente, il se développe en trois temps. On examinera d'abord l'institution du travail manuel. Il s'agit de caractériser les principaux enjeux de son introduction dans les

(1) Pascale Rougier-Pintiaux, « Les instituteurs et l'introduction du travail manuel dans les écoles primaires de garçons au XIX ${ }^{e}$ siècle ", Revue française de sociologie, XXIX, 1988, pp. 275-292; Joël Lebeaume, École, technique et travail manuel, Nice, Z'éditions, 1996 (ouvrage tiré de Cent ans de travail manuel pour l'école élémentaire. Aspects didactiques, thèse de doctorat en didactique, Université Paris-Sud, 1993). Voir également J. Lebeaume, L'Éducation technologique. Histoires et méthodes, Paris, ESF, 2000, et, dans un registre plus philosophique, Jean Houssaye, "Le travail manuel: analyseur du curriculum scolaire ", Revue française de pédagogie, $\mathrm{n}^{\circ} 132$, juillet-août-septembre 2000, pp. 67-78. 
écoles primaires (élémentaires) de garçons, ses présupposés idéologiques, mais aussi de mieux cerner quels types d'activités manuelles ses différents promoteurs veulent instituer. On considérera ensuite l'organisation de cet enseignement établi par la loi de 1882. À l'issue d'un état des lieux, tenté pour la fin de la décennie 1880, on détaillera les différentes contraintes - institutionnelles, matérielles, humaines qui pèsent sur son introduction et en particulier sur l'installation d'ateliers dans les écoles primaires. Enfin, on abordera la question plus spécifique de l'enseignement aux élèves. Se met en place, au cours de la période, une formule pédagogique cohérente qui met à distance les pratiques sociales auxquelles le travail manuel est censé se référer. Témoignage du caractère « éminemment créatif » de l'institution scolaire (1), elle résulte autant de la volonté de respecter les intentions du législateur que des contraintes évoquées ci-dessus.

\section{INSTITUER LE TRAVAIL MANUEL}

Destinée à ménager une transition en douceur entre l'école et le monde du travail, l'introduction du travail manuel à l'école primaire s'inscrit dans la problématique plus vaste d'une scolarisation - partielle ou totale - de l'apprentissage des métiers manuels (et non plus d'un accompagnement scolaire de l'apprentissage). Celle-ci trouve en grande partie son origine dans les réflexions et les débats sur l'enseignement professionnel qui accompagnent l'ouverture libérale du Second Empire et que stimule l'exposition universelle de Londres en 1862. Évoquée en 1859 par Anthime Corbon dans un livre intitulé De l'enseignement professionnel, la question de l'articulation entre enseignement général et enseignement professionnel mobilise de nombreux acteurs au début des années 1860, que ce soit au sein des commissions créées par les ministères de l'Instruction publique ou du Commerce, de sociétés comme la Société pour l'instruction élémentaire ou la Société des ingénieurs civils, ou encore de la revue L'Enseignement professionnel, fondée en 1862 (2). Mais l'attention se porte principalement sur la création d'un enseignement secondaire professionnel - ce

(1) André Chervel, «L'histoire des disciplines scolaires: réflexions sur un domaine de recherche », Histoire de l'éducation, n 38 , mai 1988, pp. 59-119.

(2) Voir notamment Robert D. Anderson, Education in France, 1848-1870, Oxford, Clarendon Press, 1975, pp. 193-224; Thérèse Charmasson, Anne-Marie Lelorrain, Yannick Ripa, L'Enseignement technique de la Révolution à nos jours. Textes officiels. Tome 1: De la Révolution à 1926, Paris, INRP/Économica, 1987, pp. 14-31. Les conceptions des ouvriers préoccupés par les questions d'enseignement 
sera l'enseignement secondaire spécial - et l'organisation de la formation ouvrière est finalement laissée à l'initiative locale.

Le mouvement est réactivé dans les années 1870. D'abord dans le contexte parisien, marqué par le mémoire d'Octave Gréard sur les écoles d'apprentis (1871) et par un rapport de Corbon «sur les exercices manuels dans les écoles primaires » (1880). Ceux-ci conduisent respectivement à des mesures de prise en charge de la formation des apprentis parisiens (parmi lesquelles la création de l'école d'apprentis du boulevard de la Villette) (1) et à l'organisation d'un enseignement manuel dans les écoles primaires de la capitale. Dans l'intervalle, un " atelier d'apprentissage »a été organisé dans une école primaire parisienne, rue Tournefort, sous le patronage actif de Gustave Salicis, délégué cantonal du cinquième arrondissement et auteur en 1875 d'un ouvrage ayant pour titre Enseignement primaire et apprentissage. Au plan national, ensuite, la question de l'enseignement manuel ressurgit dans le cadre de la préparation des grandes lois scolaires qui marquent l'avènement de la Troisième République. En marge des travaux parlementaires, la Société pour l'instruction élémentaire comme le congrès pédagogique de 1879 en font un des objets de leur réflexion (2). La loi du 11 décembre 1880 sur les écoles manuelles d'apprentissage, et celle du 28 mars 1882 qui rend l'instruction primaire obligatoire et introduit à l'école de nouvelles matières, parmi lesquelles les « travaux manuels » et l' " usage des outils des principaux métiers », sont en quelque sorte l'aboutissement de cette vaste mobilisation (3).

entre 1840 et 1870 sont analysées par Georges Duveau dans La Pensée ouvrière sur l'éducation pendant la Seconde République et le Second Empire, Paris, Domat-Monchrestien, 1948.

(1) En 1872, la municipalité décide en outre la suppression des bourses d'apprentissage et l'attribution de subventions aux « sociétés, établissements ou ateliers modèles qui contribueront à donner à l'apprentissage, dans la ville de Paris, une direction éclairée »; Délibération du conseil municipal du 27 mai 1872, in Lucien Lambeau (éd.), L'Enseignement professionnel à Paris. Premier Volume. De 1871 à 1886, Paris, Imprimerie municipale, 1898, pp. 161-162. Sur l'école du boulevard de la Villette, voir Yves Legoux, Du compagnon au technicien. L'École Diderot et l'évolution des qualifications, 1873-1972. Sociologie de l'enseignement technique français, Paris, Technique et vulgarisation, 1972.

(2) Journal d'éducation populaire, janvier-mars 1879, pp. 88-99; Émile Couturier, Les Congrès pédagogiques d'instituteurs, Paris, Imprimerie nationale, 1889.

(3) Le travail manuel est au programme des écoles primaires supérieures et des écoles normales d'instituteurs et d'institutrices dès 1881 . Contrairement à ce qu'avance 


\section{Scolariser l'apprentissage}

Les dispositifs de scolarisation du travail manuel élaborés dans les années 1860-1870 visent à résoudre ce que l'on appelle alors la « crise de l'apprentissage ». L'expression traduit le sentiment d'une dégradation des modalités traditionnelles de transmission des savoirs productifs, alors que la mécanisation accentue la division du travail: non seulement l'apprentissage en atelier prépare à un seul métier, mais il y prépare mal. La mise en concurrence de l'homme et de la machine, l'abondance de main-d'œuvre dans certains secteurs, appellent au contraire une formation polyvalente - c'est le thème de l'ouvrier complet, capable de penser et de concevoir autant que d'exécuter - qui rende possible les reconversions et permette d'éviter le chômage. La précocité de l'entrée en apprentissage, accusée de profiter davantage au patron qu'à l'apprenti, trop occupé aux menues corvées de l'atelier pour être véritablement initié au métier, est remise en question.

L'enjeu est essentiellement économique: l'affaiblissement de la valeur professionnelle des ouvriers risque de mettre en péril l'industrie nationale. Les expositions universelles, celle de Londres en 1862 comme celle de Paris en 1878, ont très certainement favorisé une prise de conscience de la nécessité d'organiser, en France, un système cohérent d'enseignement professionnel pour faire face à la concurrence étrangère. La première mission d'inspection générale relative au travail manuel, en 1882, consistera d'ailleurs à aller étudier son organisation en Suède et en Allemagne (1). À ces considérations de nature pédagogique ou technico-économique, s'ajoutent toutes sortes de motivations, comme la volonté d'éviter aux enfants et aux adolescents un contact direct avec des ouvriers soupçonnés de mœurs déréglées, ou celle de ne pas contrarier leur bon développement physique. Audelà des impératifs de la formation professionnelle, certains acteurs

P. Rougier-Pintiaux dans «Les instituteurs et l'introduction du travail manuel... », art. cit., le rapport de Corbon de 1880 n'est pas « préparatoire » à la loi du 28 mars 1882, au sens parlementaire du terme.

(1) Gustave Salicis, Guillaume Jost, De l'enseignement manuel et professionnel en Allemagne et dans les pays du Nord. $1^{\circ}$ Rapport à M. le ministre de l'Instruction publique sur une mission relative à l'enseignement du travail manuel dans divers pays étrangers; $2^{\circ}$ Notes sur quelques écoles professionnelles d'Allemagne, Paris, Delagrave, 1887; Archives nationales (AN) $\mathrm{F}^{17} 10799$, Mission des instituteurs Barthelmé et Schmitt, de Paris, et Petit, de Nancy aux écoles de travail manuel de Nääs (Suède), 1882. 
considèrent que la connaissance du travail manuel doit faire partie du bagage de tout honnête homme, envisageant même, comme Corbon, d'en étendre l'enseignement aux enfants de la bourgeoisie (1).

Dans la mesure où les écoliers du primaire sont $a$ priori destinés à travailler de leurs mains, on ne peut séparer le rejet de l'apprentissage - et plus particulièrement de l'apprentissage précoce, jugé peu formateur et peu moral - de l'hypothèse, formulée de façon prégnante dès le Second Empire, d'un enseignement primaire obligatoire. La question de l'obligation scolaire modifie en effet de façon notable les paramètres de l'éducation ouvrière des enfants et des jeunes adolescents. Pour Corbon, dont les arguments ne sont pas sans évoquer ceux qui étaient employés au XVIII ${ }^{\mathrm{e}}$ siècle pour promouvoir les écoles de dessin, l'équation est simple: dans la mesure où il faut « occuper» les enfants tout en leur évitant un placement à l'atelier, lieu de tous les dangers, ces derniers doivent rester à l'école le plus longtemps possible, jusqu'à l'âge de 15 ans (2). Mais une telle position n'invalide pas l'idée d'une familiarisation des enfants avec le travail manuel, bien au contraire. À l'école, l'enseignement manuel doit permettre de déterminer leurs aptitudes pour tel ou tel métier (ou mieux, pour une gamme de métiers) et de les préparer à recevoir un apprentissage véritablement fructueux à l'issue de leur scolarité, apprentissage qui verra sa durée raccourcie puisque les apprentis seront presque immédiatement opérationnels. C'est le sens de la création, dans les années 1860-1870, d'écoles professionnelles comme celle du boulevard de la Villette à Paris, ainsi que, en partie du moins, de la relance des écoles primaires supérieures au tournant des années 1870-1880. C'est aussi le sens de l'introduction, en 1882, du travail manuel (masculin) à l'école élémentaire, où il constitue autant une préparation à intégrer

(1) Anthime Corbon, Rapport à M. le sénateur, préfet de la Seine, sur les exercices manuels dans les écoles primaires considérés comme complément de l'éducation, Mourgues, s.d. [1880], pp. 7 et 12. Corbon propose de scolariser les fils de la bourgeoisie à l'école primaire afin de les mêler aux enfants du peuple. Voir également Henri Leneveux, Le Travail manuel en France, Paris, Librairie Germer Baillière et Cie, s.d. [vers 1881], pp. 183-184.

(2) A. Corbon, De l'enseignement professionnel, Paris, Librairie Germer Baillière et Cie, s.d. ( $4^{\mathrm{e}}$ édition), p. 129 ( $1^{\text {re }}$ édition: Dubuisson, 1859). Sur l'enseignement du dessin au $\mathrm{XVIII}^{\mathrm{e}}$ siècle, voir Renaud d'Enfert, L'Enseignement $d u$ dessin en France. Figure humaine et dessin géométrique (1750-1850), Paris, Belin, 2003; Agnès Lahalle, Les Écoles de dessin au XVIII siècle. Entre arts libéraux et arts mécaniques, Rennes, Presses universitaires de Rennes, 2006. 
directement le monde du travail qu'une propédeutique à l'école professionnelle ou primaire supérieure.

L'introduction de l'enseignement manuel à l'école est aussi un moyen de rendre plus légitime - ou plus crédible - aux yeux des familles un allongement des scolarités (jusqu'à 13 ans avec la loi du 28 mars 1882). Comme le souligne Jules Ferry à propos des élèves des écoles primaires supérieures, mais son propos pourrait tout aussi bien s'appliquer aux dernières années de la scolarité obligatoire: " ce seraient des apprentis déjà dispersés dans les ateliers, si l'école, pour les retenir, ne se transformait elle-même, dans une certaine mesure, en atelier » (1). Non seulement l'enseignement manuel apparaît comme la juste contrepartie de l'empiétement du temps des études, désormais allongé, sur la période de l'apprentissage, mais la liberté des familles désireuses d'offrir à leurs enfants une première éducation manuelle se trouve ainsi respectée. Au reste, la demande sociale à cet égard n'est pas nulle. En témoigne cette pétition parisienne «signée d'un grand nombre d'habitants du quartier de Grenelle », qui réclament la création d'une école d'apprentissage ou de cours professionnels annexés à l'une des écoles laïques de leur arrondissement (2).

\section{De la science en action}

Il faut relier l'introduction du travail manuel à l'avènement d'un enseignement de sciences spécifiquement primaire(s) dans le contexte de la rénovation pédagogique menée par les républicains. Ces derniers rêvent d'un enseignement primaire embrassant l'ensemble des connaissances humaines, théoriques et pratiques. Le travail manuel s'y inscrit d'autant plus naturellement qu'il renvoie à la destination sociale et professionnelle des élèves. L'inscription du travail manuel au nombre des applications des disciplines scientifiques enseignées à l'école primaire n'a probablement pas été assez soulignée: c'est pourtant une des dispositions de l'article $1^{\text {er }}$ de la loi sur l'instruction primaire du 28 mars 1882, selon lequel l'enseignement primaire

(1) Jules Ferry, "Rapport au président de la République française », Bulletin administratif du ministère de l'instruction publique (BAMIP), t. $24,1881\left(\mathrm{n}^{\circ}\right.$ daté du 29 octobre 1881), p. 1727.

(2) Conseil municipal de Paris, Procès-verbaux. Premier semestre 1879, Paris, Lahure, 1879, p. 219. 
comprend « les éléments des sciences naturelles, physiques et mathématiques; leurs applications à l'agriculture, à l'hygiène, aux arts industriels, travaux manuels et usage des outils des principaux métiers ». De même, l'article 80 de l'arrêté organique du 18 janvier 1887 rattache très explicitement l'enseignement du travail manuel (tout comme celui du dessin) à l'enseignement scientifique dispensé dans les écoles normales primaires. De fait, de nombreux acteurs adoptent, à des degrés divers, une approche scientiste du travail manuel que résume la formule du pédagogue Paul Robin: « la science est la théorie, le métier, l'application » (1). Pour Paul Bert, rapporteur du projet de loi sur l'instruction primaire présenté à la Chambre des députés en décembre 1879, l'enseignement scientifique ne peut être dispensé indépendamment de ses applications pratiques, qui sont intimement liées à l'industrie, à ses outils, à ses matériaux (2). Reprenant à leur compte les écrits du pasteur américain William Channing, O. Gréard et J. Ferry soulignent de la même façon les liens qui unissent le travail manuel aux applications de la mécanique et de la physique ou aux lois la nature: il n'est autre chose que la mise en œuvre des découvertes de la science, c'est de la science en action (3). On retrouve là une idée-force déjà exprimée par la revue L'Enseignement professionnel au début des années 1860 , et réactivée à partir 1876 par la revue d'orientation positiviste L'École nouvelle (4). Cette association du travail manuel et de la science traduit une certaine conception du progrès technique, fruit de la combinaison de savoir-faire pratiques

(1) Paul Robin, « L'enseignement d'un métier », cité par Christiane Demeulenaere-Douyère, Paul Robin (1837-1912). Un militant de la liberté et du bonheur, Paris, Publisud, 1994, p. 421.

(2) Article " Manuel (Travail) », in Ferdinand Buisson (dir.), Dictionnaire de pédagogie et d'instruction primaire, $1^{\mathrm{re}}$ partie, t. 2, Paris, Hachette, 1887, p. 1825.

(3) Octave Gréard, Éducation et instruction. Enseignement primaire, Paris, Hachette, 1895, pp. 336-337 (1 ${ }^{\text {re }}$ édition 1887); J. Ferry, « Discours prononcé à l'occasion de la pose solennelle de la première pierre de l'École nationale d'enseignement primaire supérieur et professionnel de Vierzon, le 3 mai 1883 », BAMIP, t. 30, pp. 184189. Gréard et Ferry (mais ils ne sont pas les seuls) s'inspirent notamment de la conférence de Channing, "De l'élévation des classes ouvrières », qu'a publiée Édouard Laboulaye dans Euvres sociales de Channing. Traduction française précédée d'un essai sur sa vie et sa doctrine, d'une introduction et de notices, Paris, G. Charpentier et Cie, 1854, pp. 97-191. Sur cette question, voir également Pierre Kahn, La Leçon de choses. Naissance de l'enseignement des sciences à l'école primaire, Lille, Septentrion, 2002, pp. 109-113.

(4) Charles Gaumont, «À nos lecteurs », L'Enseignement professionnel, 15 octobre 1862, pp. 1-2; [Gustave Francolin], « Le travail manuel », L'École nouvelle, juin 1880 , pp. 45-47. 
et d'élaborations théoriques. Parlant de l'habileté manuelle, Robin écrit: " c'est grâce à la possession de cette faculté autant qu'à des conceptions théoriques ou empiriques que les inventeurs ont fait les grandes découvertes industrielles » (1).

Cette inscription du travail manuel dans le champ des sciences appliquées, c'est-à-dire dans le domaine de la raison, a pour but de mettre le futur travailleur en état de comprendre les " principes généraux » du métier, d'en maîtriser les gestes et les matériaux, de s'adapter aux évolutions de sa profession. Elle vise également l'« ennoblissement » des professions manuelles en plaçant cet enseignement au même niveau que les disciplines traditionnelles. Pour les républicains au pouvoir, en effet, le travail est un moyen d'émancipation du peuple qui donne accès à la dignité et au bonheur (2). L'objectif est double: il s'agit de faire tomber les barrières entre activités manuelles et activités intellectuelles afin d'abolir les préjugés de classe (l'enseignement manuel est un agent de la démocratie), mais aussi de faire en sorte que les enfants des milieux populaires aiment et respectent leur métier et cherchent à y exceller, plutôt que de s'en détourner pour aller grossir les rangs des comptables, commis, et autres employés de commerce (l'enseignement manuel est un agent de la pacification sociale) (3). Enfin, la promotion du travail manuel au même rang que les sciences concourt à la réalisation du projet scolaire des républicains, qui veulent offrir aux écoliers une formation générale conjuguant éducation pratique et éducation intellectuelle et promeuvent, comme Ferry, les disciplines « accessoires » pour faire de l'école primaire une " école d'éducation libérale » développant l'ensemble des facultés (4).

(1) P. Robin, « Métiers », in F. Buisson (dir.), Dictionnaire de pédagogie..., op. cit., $2^{\mathrm{e}}$ partie, t. 2, p. 1304.

(2) Fortement teintée d'anticléricalisme, cette conception du travail nourrit l'opposition aux conservateurs catholiques. Voir par exemple le discours d'Édouard Lockroy à la Chambre des députés en 1889, publié par A. Prost dans Histoire de l'enseignement en France..., op. cit., pp. 212-213.

(3) F. Buisson, « Discours prononcé à l'occasion de la distribution des prix aux élèves de l'Association polytechnique au Cirque d'hiver, le 24 juin 1883 », Revue pédagogique, $2^{\mathrm{e}}$ semestre 1883, pp. 13-14; G. Salicis, Enseignement primaire et apprentissage, Paris, Sandoz et Fischbacher, 1875, p. 68.

(4) Discours de Jules Ferry au congrès pédagogique des instituteurs, 19 avril 1881, cité par P. Kahn, La Leçon de choses..., op. cit., p. 125. Cf. également A. Corbon, Rapport à M. le sénateur, préfet de la Seine, sur les exercices manuels..., op. cit. 
Cette articulation entre science et travail manuel est particulièrement manifeste dans l'enseignement dispensé dans le cadre des « cours normaux de travail manuel » organisés à partir de 1882 par Salicis, devenu inspecteur général, afin de former, en un an, des professeurs spécialisés pour les écoles normales et primaires supérieures. Installés rue des Ursulines à Paris, ces cours normaux seront transformés en 1884 en une École normale spéciale de travail manuel avant d'être absorbés, quelques mois plus tard (4 septembre 1884), par l'École normale supérieure d'enseignement primaire de Saint-Cloud. Les études comprennent des cours de mathématiques (géométrie et géométrie descriptive, algèbre, trigonométrie, mécanique) et de sciences (physique, chimie, histoire naturelle) ainsi que des enseignements pratiques (dessin graphique et d'ornement, modelage, travail du bois et du fer, stéréotomie), une partie de l'emploi du temps étant dévolue à l'atelier et au laboratoire. Mais au total, les sciences dominent, et moins d'un tiers du temps des études est consacré aux exercices d'atelier (16 heures hebdomadaires) (1). De façon significative, les annonces parues en 1882 et 1883 dans le Bulletin administratif du ministère de l'Instruction publique insistent bien moins sur la qualité de l'équipement des ateliers que sur la diversité des installations et des instruments scientifiques mis à la disposition des élèves: laboratoire de chimie, cabinet de physique, jardin botanique, collections, microscopes, appareils de photographie. En 1883, une vingtaine d'élèves sont même autorisés à y effectuer une seconde année d'études pour préparer le professorat des écoles normales dans l'ordre des sciences. La nature des épreuves du certificat d'aptitude à l'enseignement du travail manuel, qui sanctionne la formation, confirme cette prééminence des sciences qui tend à minorer la part de l'atelier: sur les sept épreuves en temps limité prévues par l'arrêté du 2 janvier 1885, deux seulement (modelage et exécution d'une pièce en fer ou en bois) sont consacrées au travail de la matière; et, contrairement aux épreuves de dessin et de géométrie descriptive, elles ne

(1) AN F ${ }^{17} 11633$, École normale de travail manuel, 1882-1884. À l'école d'apprentis du boulevard de la Villette, par exemple, cette proportion est comprise entre la moitié et les deux tiers. Cf. O. Gréard, Éducation et instruction..., op. cit., pp. 252-253, ainsi que Y. Legoux, Du compagnon au technicien..., op. cit., p. 116. Pour une biographie de Salicis et de ses collaborateurs René Leblanc et Gustave Philippon, voir Guy Caplat (dir.), Les Inspecteurs généraux de l'Instruction publique. Dictionnaire biographique, 1802-1914, Paris, INRP/CNRS, 1986, ainsi que Patrick Dubois, Le Dictionnaire de pédagogie et d'instruction primaire de Ferdinand Buisson. Répertoire biographique des auteurs, Paris, INRP, 2002. 
sont pas éliminatoires. Au total, il s'agit moins de posséder les rudiments des principaux métiers manuels que de maîtriser les connaissances scientifiques et techniques mobilisées par l'industrie humaine.

\section{3. Éducatif ou utilitaire?}

Deux conceptions du travail manuel et de son enseignement se développent au tournant des années 1880: d'un côté, une conception plutôt idéaliste, qui voit dans l'enseignement manuel un vecteur de rénovation sociale et lui attribue en premier lieu une fonction éducatrice; de l'autre, une conception plus pragmatique - et industrialiste qui, fortement teintée de conservatisme social, privilégie les pratiques ouvrières et l'intelligence du métier. Il faut toutefois se garder d'en donner une interprétation trop contemporaine, qui verrait là une opposition marquée entre progressistes et conservateurs, ou entre éducateurs et entrepreneurs. Certes, le débat est ouvert, par exemple entre O. Gréard et G. Salicis. Pour Gréard, l'école primaire constitue avant tout le lieu privilégié de la formation de l'intelligence et de l'acquisition durable d'un fonds de connaissance. Sa préférence va à un enseignement manuel dispensé en dehors des heures de classe, ce qui sera effectivement le cas à Paris jusqu'en 1886 (1). De son côté, Salicis, soucieux d'efficacité économique, prône une réduction des enseignements purement intellectuels au profit d'une formation manuelle adaptée au destin professionnel des enfants (2). L'atelier d'apprentissage qu'il a organisé à l'école de la rue Tournefort, où l'enseignement « tend à associer un véritable apprentissage, en vue d'une profession déterminée, aux études primaires » (3), l'atteste. Mais pour de nombreux acteurs, il n'y a pas forcément discordance entre les deux positions: elles se superposent bien plus qu'elles ne s'opposent. En témoignent les différents articles du Dictionnaire de pédagogie et d'instruction primaire consacrés au travail manuel, ou encore les discours officiels d'un Buisson ou d'un Ferry: « Former dès l'enfance l'homme et le citoyen, préparer des ouvriers pour l'ate-

(1) O. Gréard, Éducation et instruction..., op. cit., pp. 326-335; Ministère du Commerce, de 1'Industrie et des Colonies, Exposition universelle internationale de 1889 à Paris. Rapports du jury international. Groupe II. $1^{\text {re }}$ partie. Éducation et enseignement. Classes 6, 7, 8, Paris, Imprimerie nationale, 1891, p. 301.

(2) G. Salicis, Instruction pour l'enseignement manuel dans les écoles primaires, Paris, Chaix, 1885, pp. 5-8.

(3) «Mémoire de M. le préfet de la Seine au conseil municipal, 24 décembre 1883 », in L. Lambeau (éd.), L'Enseignement professionnel à Paris..., op. cit., p. 1181. 
lier, c'est notre tâche » (1), déclare ainsi ce dernier. Les programmes de 1882, qui rangent le travail manuel dans l' « éducation physique et [la] préparation à l'éducation professionnelle », sont typiques de cette superposition des deux conceptions: si l'objet de cette partie de l'enseignement est de «donner de bonne heure ces qualités d'adresse et d'agilité, cette dextérité de la main, cette promptitude et cette sûreté de mouvements $[\ldots]$ nécessaires aux élèves des écoles primaires, destinés pour la plupart à des professions manuelles », et donc de «préparer et prédisposer, en quelque sorte, les garçons aux futurs travaux de l'ouvrier », l'école ne doit pas perdre, pour autant, « son caractère essentiel d'établissement d'éducation », ni se transformer en atelier (2).

Reste à définir la place et la nature de l'enseignement du travail manuel dans le cursus primaire. Pour de nombreux acteurs, il doit faire l'objet d'une familiarisation progressive au cours de la scolarité, afin d'éviter aux enfants une rupture brutale entre l'univers de l'école et celui de l'atelier. Plus précisément, l'idée d'un enseignement manuel dispensé continûment depuis la salle d'asile (ou l'école maternelle) jusqu'à l'école primaire supérieure et/ou professionnelle, en passant par l'école primaire élémentaire, prend corps avec le rapport au ministre de l'Instruction publique sur le projet d'école nationale d'enseignement primaire supérieur et professionnel signé par Paul Jacquemart et Henri Tolain (3). Ces derniers proposent une "série ininterrompue d'exercices manuels » menant progressivement des activités de type Fröbel, qui exercent l'œil et la main des 3-6 ans, jusqu'au travail du bois et du fer en atelier, commencé à partir de 11 ou 12 ans, c'est-à-dire au cours supérieur. S'il esquisse ainsi l'enseignement de la future école nationale professionnelle de Vierzon (4), le rapport

(1) J. Ferry, « Discours... 1883 », art. cit., p. 185. Voir également F. Buisson, « Discours... 1883 », art. cit. Cf. R. d'Enfert, « "Manuel (Travail)": préparer au métier ou éduquer? », in Daniel Denis, Pierre Kahn (dir.), L'École républicaine et la question des savoirs. Enquête au cœur du Dictionnaire de pédagogie de F. Buisson, Paris, CNRS, 2003, pp. 199-222.

(2) Programmes annexés au règlement du 27 juillet 1882, BAMIP, t. 27, p. 220.

(3) « Rapport présenté au ministre de l'Instruction publique et des Beaux-Arts, au nom de la commission chargée d'élaborer un projet d'école nationale mixte d'enseignement primaire supérieur et d'enseignement professionnel », juillet 1881, BAMIP, t. 24, pp. 1131-1142.

(4) Voir également le discours prononcé le 3 octobre 1886 par le ministre René Goblet à l'inauguration de l'école professionnelle de Voiron, BAMIP, t. 40, pp. 640647. 
Jacquemart-Tolain (et le projet de programmes qui l'accompagne) préfigure également l'enseignement manuel de l'école primaire des premières décennies de la Troisième République: le programme projeté sera repris presque mot pour mot dans les arrêtés des 27 et 28 juillet 1882 réglant l'organisation pédagogique des écoles élémentaires et maternelles, puis confirmé par les règlements organiques de 1887.

Il convient toutefois de souligner la divergence entre les préconisations du rapport Jacquemart-Tolain et les conceptions de Salicis, qui sera le principal responsable de la mise en place de l'enseignement manuel dans l'instruction primaire. Ce dernier apparaît favorable au travail d'atelier à tous les niveaux de l'école primaire, comme en témoigne l'organisation de l'atelier d'apprentissage qu'il patronne rue Tournefort: prévus à l'origine (1873) pour le seul cours supérieur, qui accueille donc les élèves les plus âgés, les exercices d'atelier sont progressivement étendus au cours moyen (1877), puis au cours élémentaire (1881), l'horaire restant néanmoins proportionné à l'âge des enfants (1). Au contraire, le rapport JacquemartTolain écarte tout travail d'atelier avant 10 ans, lui préférant des exercices manuels adaptés aux jeunes enfants: " on ne doit songer qu'à développer la dextérité manuelle de l'enfant par de petits travaux n'exigeant presque aucun déploiement de force physique ». Telle sera l'orientation des programmes de 1882, qui n'introduisent le travail du bois et du fer - au sens professionnel du terme - qu'au cours supérieur, c'est-à-dire pour les élèves âgés de 11 à 13 ans, tandis que les élèves du cours moyen reçoivent simplement des «notions sur les outils les plus usuels » (2). Dominent, au cours élémentaire comme au cours moyen, les petits travaux de cartonnage, de vannerie, de fil de fer, ainsi que le modelage.

(1) G. Salicis, Instruction pour l'enseignement manuel..., op. cit., p. 6; D. Laubier, A. Bougueret, Le Travail manuel à l'école de la rue Tournefort, Paris, Hachette, 1886, p. 13.

(2) Dans certaines écoles des grandes villes (Paris, Bordeaux, Lille), les élèves seront admis à l'atelier dès le cours moyen. $C f$. Edmond Duplan, L'Enseignement primaire public à Paris, 1877-1888, Paris, Chaix, t. 1, 1889, p. 170; AN F ${ }^{17} 11705$ et 11707. À l'orphelinat de Cempuis (Oise) que dirige Paul Robin, les élèves « papillonnent » dès l'âge de 8 ans dans les différents ateliers de l'établissement; $c f$. Les Travaux manuels à l'orphelinat Prévost. Nos ateliers, le certificat d'apprentissage. L'avenir de nos enfants, Cempuis, Imprimerie de l'orphelinat Prévost, 1887. 
On mesure là, du même coup, la singularité du modèle pédagogique de l'école de la rue Tournefort. Malgré le vif intérêt qu'il suscite - l'établissement aurait reçu plusieurs centaines de visiteurs, français ou étrangers, entre 1872 et 1886 -, celui-ci ne s'accorde guère, en définitive, avec le projet scolaire des républicains. Critiqué par Gréard, mais aussi par Corbon (1), il ne parvient pas à s'imposer et demeure une expérience isolée. Jusqu'en 1887, l'école restera subventionnée par la municipalité parisienne au titre des «établissements libres d'apprentissage », tout comme d'autres ateliers d'apprentissage (typographie, ébénisterie, bijouterie-joaillerie) fondés par des industriels ou par des chambres syndicales (2).

\section{ORGANISER L'ENSEIGNEMENT MANUEL}

La décennie 1880 est celle de l'inscription du travail manuel dans le quotidien des classes. Processus de longue durée, la mise en œuvre de la loi et des programmes de 1882 demande aux différents acteurs de l'enseignement - instituteurs, inspecteurs, administrations locales, familles, éditeurs scolaires - un certain travail d'appropriation qui passe par la confrontation de leurs propres conceptions de l'enseignement manuel avec les intentions du législateur. Cet enseignement est généralement identifié au travail du bois et du fer, en référence aux écoles de type " professionnel » qui se sont développées en France depuis les années 1840, et l'attention se porte donc assez naturellement sur l'organisation des ateliers scolaires, laissant de côté - ou dans l'ombre -, dans un premier temps du moins, la mise en place d'autres formes d'enseignement manuel inscrites au programme, sur lesquelles nous reviendrons plus loin.

\section{Un état des lieux}

Établir un état des lieux de l'enseignement manuel dans les années 1880-1890, c'est-à-dire repérer où et comment cet enseignement est dispensé, mais aussi avec quelle ampleur et dans quelles

(1) AN $\mathrm{F}^{17} 11633$, Lettre de Corbon au ministre de l'Instruction publique, $1^{\text {er }}$ novembre 1885 .

(2) Voir notamment: Ville de Paris, Budget de l'exercice 1873, Paris, Mourgues, 1873, pp. 200-201. 
conditions, n'est pas chose aisée. La décennie 1880, et plus particulièrement sa première moitié, est une période de transition entre deux régimes scolaires et, loin d'être figées, les situations scolaires locales peuvent évoluer d'une année sur l'autre. Pour l'enseignement élémentaire, seul le recensement effectué par Salicis à l'occasion de l'Exposition universelle de 1889 fournit, à notre connaissance, des données chiffrées offrant une vision globale de la situation de l'enseignement manuel au niveau national (1). Outre les écoles annexées aux écoles normales d'instituteurs, il dénombre 258 écoles primaires élémentaires disposant d'un atelier, dont une centaine pour le seul département de la Seine. Dans le même temps, Salicis estime à 12000 environ le nombre d'écoles de garçons ou mixtes proposant un enseignement manuel "sans atelier », soit un peu plus d'une sur quatre (2): estimation qui reste à confirmer dans la mesure où elle est inférée à partir de données recueillies pour trois départements seulement. Des monographies, récentes ou plus anciennes, des rapports annuels d'inspecteurs d'académie, des procès-verbaux de conseils généraux permettent d'affiner ce panorama national trop grossier en resserrant la focale au niveau des départements. Dans plusieurs d'entre eux, aucune école élémentaire ne dispose d'un atelier ou de l'outillage nécessaire à la fin de la décennie 1880. La Corse, la HauteSaône, la Saône-et-Loire, la Seine-Maritime sont dans ce cas vers 1887-1888 (3). Dans certains départements, un enseignement manuel est attesté, mais on ne connaît que rarement sa nature (avec ou sans atelier), la proportion d'écoles concernées et les caractéristiques de ces dernières (4). Si la rareté des ateliers scolaires semble bien réelle - vers 1885, le département du Doubs, étudié par Jacques Gavoille, n'en compte que quatre, implantés dans des écoles comprenant un cours complémentaire -, elle n'interdit pas l'existence d'autres types d'activités manuelles telles que vannerie, réalisation de solides géo-

(1) G. Salicis, Enseignement du travail manuel, Paris, Imprimerie nationale, 1889. L'auteur évalue à 180 le nombre d'écoles normales et d'écoles annexes, et à 211 le nombre d'écoles primaires supérieures et de cours complémentaires dotés d'un atelier.

(2) Le nombre des écoles de garçons ou mixtes est alors d'environ 43000. $C f$. Jean-Pierre Briand et al., L'Enseignement primaire et ses extensions, XIXe-XXe siècles. Annuaire statistique, Paris, INRP/Économica, 1987, pp. 162-163. 1888.

(3) AN $F^{17} 9675$, Organisation du travail manuel dans les écoles normales, 1886-

(4) Situation scolaire des départements en 1878 et en 1888, Paris, Imprimerie nationale, 1889. 
métriques en carton, en bois ou en terre glaise, fabrication au couteau d'instruments aratoires en miniature (1). Enfin, sur les 30 villes les plus importantes du pays, 10 seulement, d'après le recensement de Salicis, proposent un enseignement manuel aux élèves de leurs écoles élémentaires, dont 7 des travaux d'atelier: Paris, Lyon (en projet), Marseille (5 écoles seulement), Bordeaux, Lille, Montpellier, Tourcoing. Manquent à l'appel des villes comme Nantes, Rouen, Nancy. Contrairement à une idée répandue, les grandes agglomérations ne disposent pas toujours de l'offre la plus développée.

L'enseignement post-élémentaire est mieux documenté, quantitativement et qualitativement. Pour l'enseignement primaire supérieur, les réponses à la grande enquête de novembre 1886 sur l'enseignement technique et professionnel, menée conjointement par le ministère de l'Instruction publique et le ministère du Commerce et de l'Industrie (2), fournissent de nombreuses informations sur l'enseignement manuel dispensé: programme suivi, horaires hebdomadaires, existence (ou non) d'ateliers, profil des maîtres, finalités. Le cas des cours complémentaires, qui proposent, au sein des écoles élémentaires, des poursuites d'études en un ou deux ans (parfois plus) après l'obtention du certificat d'études primaires, permet tout particulièrement d'éclairer une réalité difficile à saisir.

Sur 278 cours complémentaires de garçons répartis dans 75 départements (3), moins d'un établissement sur deux (120, soit $43 \%$ ) inscrit le travail manuel à son programme. Il est vrai que la réglementation de 1881, qui institue les cours complémentaires, passe cet enseignement sous silence, et qu'il n'est explicitement inscrit à leur plan d'études qu'à partir de 1885 (avec le même programme que celui des écoles primaires supérieures). En outre, certains cours de

(1) «Les travaux manuels à l'école primaire », Revue pédagogique, $2^{\mathrm{e}}$ semestre 1883, pp. 81-82; Jacques Gavoille, L'École publique dans le département du Doubs (1870-1914), Paris, Les Belles Lettres, 1981, p. 288. Voir également Jean-Louis Clade, École et instituteurs dans le Jura, Yens-sur-Morges, Cabédita, 2002, p. 140.

(2) Circulaire du 22 novembre 1886, BAMIP, t. 40, pp. 1041-1042. La circulaire et le questionnaire qui l'accompagne sont publiés dans T. Charmasson et al., L'Enseignement technique..., op. cit., pp. 284-290.

(3) $\mathrm{AN} \mathrm{F}^{17} 11707$ et 11705 , Réponses à la circulaire ministérielle du 22 novembre 1886. Plusieurs départements, dont le département de la Seine, sont manquants. La Statistique de l'enseignement primaire supérieur (écoles et élèves) au 31 décembre 1887, Paris, Imprimerie nationale, 1888, recense 308 cours complémentaires de garçons. 
création récente ne sont pas complètement organisés au moment de l'enquête. Si l'offre d'enseignement manuel apparaît globalement plus développée dans les départements à forte densité de population industrielle (1), en revanche, l'importance démographique de la ville d'implantation comme l'effectif de l'école de rattachement ne jouent pas significativement en sa faveur. Le travail manuel est enseigné à Forcalquier (3000 habitants) dans une école de 48 élèves (dont 8 au cours complémentaire), tout comme à Blamont (650 habitants) - petit bourg situé, il est vrai, dans la région industrielle de Montbéliard -, mais pas à Lens (12000 habitants), où le cours complémentaire (82 élèves) est annexé à une école de 7 classes (500 élèves), ni même à Toulouse, sixième ville du pays par sa population (140000 habitants). L'effectif des cours complémentaires proprement dits n'apparaît pas non plus décisif: environ $46 \%$ des « gros » cours (plus de 20 élèves) y initient leurs élèves, contre $40 \%$ pour ceux à petit effectif (moins de 20 élèves). Quant aux ateliers, une cinquantaine de cours complémentaires seulement en disposent, parfois mal outillés: l'enseignement manuel y est plus souvent assuré par des maîtres-ouvriers - menuisier pour le travail du bois, serrurier ou mécanicien, voire ingénieur, pour le travail du fer - que par le directeur ou un maîtreadjoint, mais on peut aussi rencontrer des solutions mixtes, comme à Pont-l'Évêque où le directeur et un professeur spécial se partagent l'enseignement en atelier.

Il faut souligner l'existence de formules de substitution dans les cours complémentaires qui ne disposent pas d'atelier. Une première solution réside dans l'organisation d'activités manuelles ne nécessitant pas ou peu d'outillage, peu différentes, finalement, de ce qui peut se pratiquer à l'école élémentaire. Une seconde solution, assez répandue, consiste à conduire les élèves chez un serrurier ou un menuisier de la ville (ce qui ne veut pas dire que le directeur du cours perd tout contrôle sur cet enseignement); il peut également leur être demandé, comme au Pouzin (Ardèche), de « présenter chaque semaine un travail exécuté à domicile sur les indications et d'après un croquis donnés par le maître ». Un système un peu différent sera mis en place à Creil à partir de 1887: les élèves du cours complémentaire sont

(1) Les quinze départements en tête sur ce critère concentrent la moitié de l'offre. Estimation d'après la Statistique générale de la France. Résultats statistiques du dénombrement de 1886. Première partie: France, Paris, Berger-Levrault, 1888, pp. 135-136. 
accueillis, à la belle saison et à raison de trois demi-journées par semaine, dans une école gratuite de travail manuel (avec atelier et musée industriel) fondée à leur intention par un industriel de la ville (1). Mais, comme le révèle l'enquête de 1886, cette « externalisation » de l'enseignement manuel se ramène parfois à un système d'alternance école/atelier relativement proche des formes traditionnelles de l'apprentissage, que ce soit chez un professionnel ou dans le cadre familial. C'est ainsi que les élèves du cours complémentaire de Casteljaloux (Lot-et-Garonne), pourvu qu'ils soient titulaires du certificat d'études, «vont travailler 3 jours par semaine, de 5 heures à 7 heures du soir, dans un atelier de leur choix », le directeur de l'école se rendant chez les patrons « pour examiner le travail et prendre des notes ». À Hyères, où l'enseignement manuel n'est pas organisé, « les élèves qui en font la demande sont envoyés en ville chez un maître serrurier désigné par la municipalité ». En revanche, les enfants de Rougemont (Haut-Rhin) sont simplement « initiés à ces travaux par leurs parents qui sont presque tous occupés dans les ateliers ». Reste à savoir si de telles pratiques visent à pallier les carences de l'offre scolaire ou si, implantées de longue date, elles jouent en défaveur de l'institution de formes plus scolaires d'enseignement manuel. Du moins est-il possible de préciser les contraintes, institutionnelles, humaines et matérielles, qui pèsent sur l'introduction du travail manuel au cours de la période.

\section{Une institutionnalisation a minima?}

L'introduction du travail manuel bute sur la question de son institutionnalisation et de sa légitimité au regard des autres disciplines de l'école, mieux implantées parce que plus anciennes. Disposant d'un horaire important dès l'école élémentaire (2 à 3 heures par semaine) et d'une réglementation favorable (un atelier doit être créé dans toute nouvelle école) (2), le travail manuel n'est en revanche guère valorisé

(1) Henri Doliveux, "Une école de travail manuel et un patronage modèles », Revue pédagogique, $2^{\mathrm{e}}$ semestre 1897, pp. 27-35. Voir également J.-P. Briand, J.-M. Chapoulie, Les Collèges du peuple. L'enseignement primaire supérieur et le développement de la scolarisation prolongée sous la Troisième République, Paris, INRP/CNRS/Presses de l'ENS, pp. 212-213.

(2) Instruction spéciale du 28 juillet 1882, BAMIP, t. 27, pp. 430-438. Dans les écoles de moins de trois classes, l'atelier peut être aménagé dans le préau. Cette instruction reprend, de façon moins contraignante, un arrêté du 17 juin 1880 . Un décret du 29 janvier 1890 relatif au matériel obligatoire (BAMIP, t. 47, pp. 149-154) en réduira encore la portée. 
par les examens, dont on sait qu'ils jouent un rôle essentiel dans le mécanisme de légitimation d'une discipline scolaire. Ni l'examen du certificat d'études primaires élémentaires, qui sanctionne la scolarité élémentaire, ni ceux des brevets de capacité (brevet simple et brevet supérieur), qui scandent la formation des instituteurs, ne comportent d'épreuve de travail manuel. La différence de traitement entre garçons et filles doit être signalée: si les candidates au certificat d'études primaires ou au brevet élémentaire sont soumises à une épreuve de couture (dont l'enseignement relève d'une tradition plus ancienne), il n'existe pas de disposition équivalente pour les candidats. Évoquée lors du congrès des instituteurs de 1887, l'hypothèse d'une inscription du travail manuel masculin au certificat d'études primaires est rejetée faute d'un accord sur les frontières de cet enseignement (1). Au total, seul le certificat d'études primaires supérieures, institué en 1882, comporte une épreuve de travail manuel, mais les conditions matérielles nécessaires à sa bonne tenue (atelier, outillage) ne sont pas toujours réunies (2). On peut dès lors se demander si cette quasiabsence de sanction par l'examen n'a pas contrarié la reconnaissance de l'enseignement manuel comme discipline à part entière, tant par les instituteurs que par les élèves et leurs familles. Même le certificat d'aptitude à l'enseignement du travail manuel a vu un moment son existence menacée, après l'absorption de l'école normale du travail manuel par celle de Saint-Cloud. Supprimé en juillet 1885, il est finalement rétabli par l'arrêté organique du 18 janvier 1887.

L'absence de doctrine pédagogique clairement explicitée par les instances officielles doit également être soulignée. Si, pour l'enseignement élémentaire, un programme de travail manuel est arrêté dès

(1) Une épreuve facultative est néanmoins envisagée. $C f$. Congrès des instituteurs et des institutrices de France, 4-8 septembre 1887. Compte rendu des travaux, $2^{e}$ fascicule. Procès-verbaux des séances générales. $2^{e}$ session, Paris, Au siège du Congrès, 1887, p. 143. En 1890, le congrès de la Ligue de l'enseignement demande que l'enseignement manuel reçoive une "sanction efficace » à l'examen du certificat d'études; cf. L'Enseignement manuel et expérimental, mai 1890, p. 115. Il faut attendre un arrêté du 19 juillet 1917 pour que soit instituée, pour les garçons seulement, une épreuve du certificat d'études primaires élémentaires comportant « un exercice très simple de dessin linéaire ou d'ornement (ou un exercice de travail manuel) », BAMIP, t. 102, pp. 231-235.

(2) Circulaire du 10 mai 1884, BAMIP, t. 34, p. 194. Sur le certificat d'études primaires supérieures qui, en outre, n'a guère de valeur d'usage, voir Patrick Cabanel, La République du certificat d'études. Histoire et anthropologie d'un examen (XIX ${ }^{e}-X X^{e}$ siècles), Paris, Belin, 2002, pp. 86-88. 
1882, il faut attendre 1885 pour que celui de l'enseignement primaire supérieur soit fixé (arrêté du 27 juillet) (1), et 1891 pour les écoles normales primaires (arrêté du 3 janvier). Ces dernières, qui constituent pourtant un lieu privilégié pour initier les instituteurs au travail manuel et à son enseignement, restent en outre mal équipées avant 1890, faute d'investissements suffisants (2). Mais surtout, les maitres, qui ne peuvent guère s'appuyer sur une quelconque expérience antérieure, ne disposent pas de directives pédagogiques avant le milieu de la décennie. Le congrès international des instituteurs du Havre de 1885 fait ainsi ressortir la nécessité d'élaborer ce que Charles Defodon appelle une «méthode pratique d'enseignement manuel »on dirait aujourd'hui une didactique du travail manuel - qui soit aisément applicable dans l'ensemble des écoles (3). C'est très certainement cette prise de conscience collective qui conduit le ministre René Goblet à se préoccuper de la formation des maîtres - « il importe [...] que l'école normale donne à l'enseignement manuel des règles, des principes, une méthode, une direction » - et l'inspecteur général Salicis à publier successivement, en 1885 et 1886, deux instructions fournissant directions pédagogiques et conseils pratiques (4).

L'institutionnalisation d'une discipline ne se mesure pas seulement à l'aune des prescriptions officielles. Elle s'apprécie également à la façon dont les acteurs de l'école s'en emparent et travaillent à sa mise en forme et à sa popularisation. Parmi ces derniers, les organes de diffusion que sont les manuels scolaires et la presse pédagogique adoptent le plus souvent une position d'attente jusqu'au milieu de la décennie. Si l'édition scolaire reste d'abord silencieuse, c'est qu'elle doit faire face à un véritable vide didactique, lié à l'absence de définition claire des contours de la discipline. Les premiers livres scolaires dédiés au travail manuel masculin ne sont publiés qu'à partir de 1886,

(1) L'arrêté du 15 janvier 1881 mentionne simplement: «Fer, bois, jardinage, etc. ».

(2) Un rapport de Salicis du 29 mai 1886 (AN F ${ }^{179674)}$ estime à 600000 francs la somme nécessaire à l'organisation du travail manuel dans les 89 écoles normales d'instituteurs, et à 350000 francs la dépense annuelle engendrée par cet enseignement.

(3) Charles Defodon, « Les travaux du congrès du Havre. Questions sur le travail manuel », Manuel général de l'instruction primaire, 1885, partie générale, pp. 462463.

(4) Circulaire du 28 octobre 1885, BAMIP, t. 38, pp. 876-880; G. Salicis, Instruction pour l'enseignement manuel..., op. cit.; idem, Instruction spéciale sur l'enseignement du travail manuel dans les écoles normales d'instituteurs et les écoles primaires, élémentaires et supérieures, Paris, Imprimerie nationale, 1886. 
en faible nombre cependant: moins d'une dizaine de manuels entre 1886 et 1890, une trentaine dans la décennie 1890 (en comptant les rééditions), d'après le catalogue informatisé de la Bibliothèque nationale de France (1). De même, une publication aussi emblématique que le Dictionnaire de pédagogie et d'instruction primaire de Ferdinand Buisson, qui paraît dans la même période, ne manifeste guère d'engagement pédagogique malgré un discours idéologique vigoureux sur les finalités de la discipline: sauf à compter l'article " Métier », signé par Paul Robin, aucun article relatif au travail manuel ne figure dans la deuxième partie de l'ouvrage, pourtant censée former un « dictionnaire de leçons » à l'usage des instituteurs.

L'examen de la presse d'éducation invite toutefois à nuancer le propos. Certes, la Revue pédagogique, dont l'une des fonctions est de diffuser les conceptions officielles auprès des cadres de l'instruction primaire, ne se risque guère sur le terrain de la réflexion pédagogique. En 1883 et 1884, par exemple, elle se borne à publier des témoignages d'acteurs de terrain - instituteurs ou inspecteurs -, lesquels semblent parfois aller à l'encontre de la volonté officielle de scolarisation du travail manuel, tel cet instituteur qui a fait réaliser à ses élèves des objets «à la maison » au prétexte que les familles rurales possèdent «quelques outils élémentaires » (2). Mais il est des périodiques qui s'impliquent tôt dans la recherche de solutions pédagogiques, peut-être parce qu'ils sont plus proches de la base et plus sensibles aux demandes ou aux interrogations des instituteurs. Dès 1883, la revue L'Instruction primaire fournit des indications pratiques en vue d'une mise en œuvre effective du programme des écoles primaires de garçons (3). L'inclusion de leçons de travail manuel dans les rubriques « scolaires » de ces revues reste cependant tardive: 1885 pour le Manuel général de l'instruction primaire, 1886 pour L'Instruction primaire, soit environ deux ans après l'introduction du travail manuel féminin dans ces mêmes rubriques (4). En

(1) Selon la même source, plus de 220 manuels de dessin sont publiés entre 1880 et 1900 .

(2) Revue pédagogique, $1^{\mathrm{er}}$ semestre 1884, pp. 488-489. Voir également Revue pédagogique, $2^{\mathrm{e}}$ semestre 1883, pp. 81-82 et 284-285.

(3) C.G. [Claude Georgin], « Les nouveaux programmes de l'enseignement primaire », L'Instruction primaire, 1882-1883, pp. 660-662, 718-720, 742-743, 830-831, 851-853 et 871-874.

(4) Dès 1880 , la revue L'École nouvelle publie des leçons de travail manuel faisant partie d'un « cours d'études intégrales ». Les leçons du Manuel général, signées 
1900, l'inspecteur général René Leblanc constatera encore l'absence des exercices manuels dans une partie de la presse pédagogique (1).

\section{Des acteurs peu convaincus}

Il faut également tenir compte des réticences, voire des résistances à l'introduction du travail manuel. D'abord, son utilité n'est pas unanimement reconnue, spécialement dans les régions rurales où, bien souvent, les élèves rejoignent l'exploitation familiale à l'issue de leur scolarité. À Chabeuil (Drôme), le travail manuel n'est pas enseigné au cours complémentaire, car la population est « presque entièrement agricole »; à Saint-Nicolas-de-la-Grave (Tarn-et-Garonne), il est remplacé par des promenades et des travaux agricoles (2). Les familles, et plus généralement la « demande sociale », attendent surtout de l'école qu'elle se consacre aux apprentissages fondamentaux - orthographe, grammaire, calcul, géométrie - et que les enfants ne perdent pas leur temps avec des exercices manuels. Dans le département du Doubs, les parents font même de la résistance, refusant d'envoyer leurs enfants suivre des leçons de travail manuel dont ils ne perçoivent guère l'utilité (3). Chez les élites locales, mais aussi chez les instituteurs, ces réserves peuvent prendre une tournure plus idéologique: utile, peut-être, dans les villes, l'enseignement manuel risque d'amplifier l'exode rural en détournant les enfants des campagnes du « milieu naturel où ils sont appelés à vivre » (4). Par ailleurs, l'impression d'une trop grande proximité avec la formation professionnelle suscite une certaine hostilité des instituteurs. Leurs

Émile Faivre, sont publiées en 1887 par Hachette sous le titre Enseignement du travail manuel à l'école primaire. Exercices gradués conformes aux programmes officiels.

(1) Ministère du Commerce, de l'Industrie, des Postes et des Télégraphes, Exposition universelle internationale de 1900 à Paris. Rapports du jury international. Groupe I. Éducation et enseignement. Première partie. Classe 1, Paris, Imprimerie nationale, 1902, p. 992. Une cinquantaine de revues évoquant le travail manuel dans le cadre de l'école, mais aussi de l'éducation familiale, sont recensées dans Pierre Caspard (dir.), La Presse d'éducation et d'enseignement, XVIII' siècle-1940. Répertoire analytique, Paris, INRP/CNRS, 1981-1991. La moitié d'entre elles paraissent entre 1876 et 1914 (ou au-delà).

(2) $\mathrm{AN} \mathrm{F}^{17} 11707$.

(3) J. Gavoille, L'École publique dans le département du Doubs..., op. cit.,

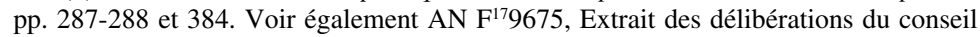
général d'Indre-et-Loire, séance du 22 avril 1888.

(4) C. Defodon, « Les travaux du congrès du Havre... », art. cit., p. 463. Cf. également AN F ${ }^{17} 9675$, Extrait des délibérations du conseil général de l'Yonne, séance du 23 août $1888 ; i d$. de la Creuse, séance du 19 avril 1887. 
contributions au congrès international de l'enseignement primaire de 1889 montrent en effet que les travaux d'atelier, assimilés à un enseignement professionnel (et accusés de " surcharger » les programmes), leur semblent incompatibles avec une instruction primaire élémentaire censée être entièrement consacrée à l'éducation générale. Certains instituteurs, comme ceux du département de la Seine, demandent même leur suppression pure et simple (1).

Les réticences des instituteurs à l'égard du travail manuel trouvent également leur traduction dans l'échec presque complet des cours d'été organisés à leur intention par le ministère de l'Instruction publique en 1887 et 1888. Réclamés au congrès du Havre, ces cours d'une durée de quatre semaines n'ont pu avoir lieu que dans quatre écoles normales en 1887, dans dix seulement l'année suivante (2). Ils seront ensuite supprimés. Si une part de responsabilité dans cet échec est à imputer au ministère de l'Instruction publique, qui limite sa participation financière, ainsi qu' aux conseils généraux, qui refusent très majoritairement la subvention nécessaire à leur tenue, l'insuccès de ces stages tient aussi à la faible mobilisation des maîtres. Dans de nombreux départements, le nombre insuffisant d'inscrits (parfois inférieur à la dizaine, voire nul) entraîne leur annulation, justifiant $a$ posteriori les réserves des assemblées départementales qui y voient la preuve de leur inutilité. Probablement les instituteurs n'étaient-ils pas prêts à sacrifier une partie de leurs vacances et de leur traitement - le stage à l'école normale signifie éloignement du domicile et sacrifices financiers, les stagiaires devant s'acquitter des frais de leur séjour pour aller suivre une formation dont ils ne percevaient guère l'intérêt, faute généralement de disposer des équipements nécessaires dans leur école.

\section{Des obstacles financiers}

Le développement de l'enseignement manuel est très directement confronté à des questions d'ordre financier. On l'a dit, cet enseigne-

(1) Congrès international de l'enseignement primaire, Paris, Delagrave/Hachette, 1889, pp. 1-63; "Le congrès international de l'enseignement primaire de 1889 », L'Instruction primaire, 1889-1890, pp. 2-4. Voir également, sur le même thème, les délibérations des conseils généraux dans AN F ${ }^{17} 9675$.

(2) AN F179675, Notes à Gustave Salicis des 13 août 1887 et 22 septembre 1888. Voir également les cartes établies par Joël Lebeaume dans sa thèse, pp. 134 bis et 135 bis. 
ment est généralement identifié au travail du fer et du bois; c'est donc autour de son organisation matérielle - en fait l'installation de l'atelier scolaire - que se cristallisent les questions relatives à sa mise en place. L'absence d'atelier ou le manque d'outils fait souvent partie des arguments utilisés, au milieu de la décennie 1880, pour justifier l'absence de tout enseignement manuel dans les écoles. À l'inverse, la réussite de la nouvelle discipline apparaît, aux yeux des protagonistes, étroitement liée à la qualité des conditions matérielles de son enseignement. Dans le contexte du fonctionnement largement décentralisé de l'institution primaire, l'absence d'un véritable engagement financier de l'État pour pallier les carences des collectivités locales la gratuité et l'obligation scolaires appellent d'autres priorités budgétaires -, a constitué un frein puissant à la popularisation du travail manuel, non seulement au niveau de la formation des maîtres, comme on l'a brièvement évoqué plus haut, mais aussi au niveau des écoles élémentaires.

Installer et équiper un atelier dans une école coûte cher. Selon l'Instruction de 1885, l'acquisition du matériel nécessaire aux travaux de menuiserie, ajustage, tour, forge et modelage, revient à 2000 francs environ pour une école de 100 élèves (1). Somme à laquelle viendront s'ajouter annuellement les dépenses liées à l'achat des matériaux consommables (200 francs) ainsi qu'à l'entretien des outils et à la rémunération éventuelle d'un maître-ouvrier. Outre le fait que l'école doit disposer d'un local approprié, l'atelier scolaire représente donc une charge importante pour les municipalités, dont dépendent financièrement les écoles élémentaires. L'inspecteur primaire de la circonscription de Pontoise estime ainsi que le travail du bois et du fer « exigerait de la part des communes des dépenses qui seraient, dans la plupart des cas, incompatibles avec leurs ressources » (2). D'autant que, à bien lire la réglementation officielle, le maniement des outils ne concerne $a$ priori que les écoliers inscrits au cours supérieur ou au cours complémentaire. Or, quand ils existent, les cours supérieurs n'accueillent généralement qu'une minorité d'élèves, ce qui n'incite guère à la dépense. Si des ateliers sont aménagés préférentiellement dans les écoles urbaines, ce n'est donc pas

(1) G. Salicis, Instruction pour l'enseignement manuel..., op. cit., pp. 24-31.

(2) Musée départemental de l'éducation de Saint-Ouen-l'Aumône, Conférence pédagogique du 27 mars 1889 sur les essais de travail manuel dans l'école primaire élémentaire (canton de Montmorency). 
seulement parce que les industries se développent dans les villes et leurs faubourgs. C'est aussi parce que, à la ville plus qu'en milieu rural, le coût de leur installation n'apparaît pas trop disproportionné par rapport aux effectifs scolaires. Reste que la création d'un atelier ne débouche pas toujours sur son emploi effectif. À Lyon, par exemple, les ateliers des groupes scolaires nouvellement construits étaient toujours inutilisés à la fin de la décennie 1880 (1).

Deux solutions sont alors possibles pour organiser des travaux d'atelier dans les écoles élémentaires. La première consiste à exiger, de façon autoritaire, leur financement par les municipalités. Une note adressée au directeur de l'enseignement primaire suggère une « imposition d'office » pour les communes qui n'auraient pas doté leurs écoles de l'outillage nécessaire à l'enseignement manuel (2): idée aussitôt repoussée, car elle les obligerait à des dépenses supplémentaires. Certains instituteurs n'y auraient pourtant pas été hostiles, tels ceux du département de l'Aude qui, à l'occasion du congrès de 1889, demandent que les achats de matériel soient obligatoirement inscrits aux budgets municipaux (3). La seconde solution s'accorde mieux, en réalité, avec un certain libéralisme républicain. Elle consiste à faire appel à l'initiative individuelle, celle des maîtres comme celle de personnes privées, pour compléter, voire suppléer l'action de l'État ou des collectivités locales. À la grande satisfaction des autorités, des instituteurs entreprenants équipent eux-mêmes tout ou partie des ateliers, parfois à leurs frais. La Revue pédagogique signale notamment ces instituteurs de l'Aisne, " aussi dévoués qu'ingénieux », qui « se sont mis courageusement à l'œuvre et n'ont pas attendu que l'école fût dotée d'un outillage complet pour se

(1) J. Janicot, Monographie des écoles communales de Lyon depuis 1828 jusqu'en 1891 et la construction des groupes scolaires, Lyon, Librairie Joseph Chanard, 1891, p. 61. Voir également Jean-François Martin, Marie-Françoise Rosset, L'École primaire dans le Rhône (1815-1940), Lyon, Conseil général du Rhône, Archives départementales, Service éducatif, 1997. Selon ces derniers, «les communes, les inspecteurs d'académie, les architectes jouissent d'une certaine liberté pour fixer au cas par cas le programme architectural » (p. 154).

(2) AN F179675, Note anonyme du 15 mai 1888. Voir également (même cote) le rapport de l'inspecteur général Gustave Philippon " sur les cours normaux de vacances », s.d. (octobre 1887), dans lequel celui-ci souhaite que les préfets fassent pression sur les maires pour qu'un crédit spécifique soit inscrit aux budgets municipaux.

(3) Congrès international de l'enseignement primaire, op. cit., p. 7. 
conformer à l'arrêté du 27 juillet » (1). Également sollicités, les soutiens privés prennent des formes multiples: dons de matériel, comme à Roquevaire (Bouches-du-Rhône), où les cercles républicains ont complété l'outillage, participation à titre gracieux d'ouvriers ou d'artisans locaux, ou encore investissement personnel d'un industriel, comme à Creil, dans l'Oise, ou à Hérimoncourt, dans le Doubs. Dans ce dernier cas, la maison Peugeot frères fournit pour 200 francs d'outils au cours complémentaire et y envoie gratuitement un de ses ouvriers pour s'occuper de l'atelier de menuiserie (2).

Dans ce contexte, la capitale confirme sa singularité par rapport au reste du pays. À Paris, où plus d'une centaine d'écoles sont pourvues d'un atelier vers 1890 , les sommes consacrées à l'enseignement manuel sont sans commune mesure avec celles qui ont été évoquées ci-dessus. Il est vrai que le nombre de petits Parisiens scolarisés est considérable (environ 65000 garçons, dont un quart environ prend part aux exercices d'atelier) (3) et que la municipalité dispose de ressources financières qui n'ont pas d'équivalent ailleurs. Après une forte croissance, qui correspond à la multiplication des équipements, la dépense municipale annuelle en faveur du travail manuel dépasse les 400000 francs à partir de 1884, l'essentiel allant au traitement de quelque 200 maîtres-ouvriers. À partir de 1892, une réorganisation de l'enseignement manuel permet de réduire significativement les crédits alloués: la direction de l'enseignement manuel est confiée aux instituteurs (un cours normal est d'ailleurs créé à leur intention) et le nombre de maîtres-ouvriers divisé par trois (4).

(1) «Les travaux manuels à l'école primaire ", Revue pédagogique, $2^{\mathrm{e}}$ semestre 1883, p. 82. Voir également G. Salicis, Enseignement du travail manuel, op. cit., pp. 58, 61, 64 et 66 .

(2) AN $\mathrm{F}^{17} 11707$. $C f$. également "L'atelier manuel d'apprentissage de l'école publique de Roquevaire », BAMIP, t. 26, 1882 (n daté du 13 mai 1882), p. 298; H. Doliveux, « Une école de travail manuel... », art. cit.

(3) Exposition universelle internationale de 1889..., op. cit., p. 359.

(4) Compte général des recettes et dépenses de la ville de Paris, Paris, Paul Dupont, publié annuellement; Conseil municipal de Paris, Procès-verbaux. Deuxième semestre 1891, op. cit., pp. 868-873. 


\section{ENSEIGNER LE TRAVAIL MANUEL}

Considérés dans un premier temps comme une pièce essentielle du dispositif d'enseignement du travail manuel, les exercices d'atelier voient leur place - et leur fonction - revue à la baisse dès la fin des années 1880. Il s'agit de tenir compte de réalités et de contraintes aussi diverses que l'environnement immédiat (urbain ou rural, industriel ou agricole), la structure pédagogique et les ressources matérielles des écoles, les compétences des maîtres ou encore la demande des familles. Il s'agit également de réduire la « surcharge » des programmes due à la multiplication des matières depuis 1882, et surtout de maintenir l'école élémentaire dans ce que les instituteurs considèrent comme sa finalité première, à savoir dispenser un enseignement général sans préjuger outre mesure de l'avenir professionnel des élèves.

\section{L'atelier contesté}

Mais c'est aussi le principe même de l'atelier scolaire qui est contesté, y compris par les deux principaux collaborateurs de Salicis, Gustave Philippon et René Leblanc, qui ont théoriquement pour charge de le promouvoir. Premier reproche: les travaux d'atelier ne sont pas toujours en rapport avec la stature ou la force musculaire des enfants, au risque de porter préjudice à leur bon développement physique. Initialement utilisés en faveur de la scolarisation du travail manuel, les arguments hygiénistes sont retournés contre les exercices d'atelier, jugés encore trop précoces, même encadrés par l'école. Pour l'inspecteur général Félix Martel, «il n'est pas bon [...] de mettre à l'établi ou à l'étau des enfants de sept à dix ans et de les exercer à jouer à l'ouvrier, comme, il n'y a pas longtemps encore, on les habituait avec les bataillons scolaires à jouer au soldat » (1). L'âge de dix ans semble parfois encore trop jeune pour accéder à l'atelier, et la barre peut être rehaussée à douze ans, âge qui correspond approximativement au terme de la scolarité élémentaire (2). En second lieu,

(1) Félix Martel, «Le travail manuel éducatif et l'apprentissage à l'école », Revue pédagogique, $2^{\mathrm{e}}$ semestre 1891, p. 147. Celui-ci craint également qu'une trop grande place accordée aux exercices d'atelier dans les écoles primaires supérieures ne détourne les familles vers des établissements privés offrant un enseignement général plus développé.

(2) René Leblanc, «Commentaire du programme des travaux manuels pour les écoles normales d'instituteurs », Revue pédagogique, $1^{\text {er }}$ semestre 1891, p. 234. 
la participation des maitres-ouvriers est largement remise en question, les instituteurs réclamant, contre ces derniers, l'exclusivité de l'enseignement manuel (1). Praticiens, mais non éducateurs, les ouvriers n'auraient pas toujours les compétences pédagogiques et les qualités morales que l'on attend d'un enseignant; ils seraient, en outre, inaptes à dispenser un enseignement raisonné du travail manuel, se bornant à transmettre des tours de main faute de connaissances théoriques suffisantes: "ils cherchent, affirme Philippon, à faire immédiatement de l'utile quand nous cherchons surtout à faire de l'éducation » (2). De son côté, Leblanc signale leur incapacité à donner la « raison scientifique » des gestes techniques, qu'il s'agisse d'un tracé ou du maniement d'un outil (3). Enfin, cette défaveur pour les travaux d'atelier débouche sur une remise en cause de la formation des instituteurs. Celle-ci leur accorderait une place trop exclusive au détriment des travaux manuels élémentaires tels qu'ils peuvent et doivent - se pratiquer dans une école ordinaire (4).

Il ne fait guère de doute que cette critique a pour but de bien marquer la frontière entre l'école et l'atelier, entre l'éducation et l'apprentissage. Jointe à la prise en compte des contraintes matérielles évoquées plus haut, elle conduit à une réévaluation de l'enseignement manuel visant à inclure celui-ci dans l'ordinaire des classes en accordant ses finalités, ses contenus et ses méthodes aux normes de l'école. Cette réévaluation est essentiellement fondée sur une lecture plus littérale - et qui se veut plus respectueuse des intentions initiales - des programmes officiels. Dans la deuxième moitié de la décennie 1880, Leblanc et Philippon développent la notion de travail manuel « élémentaire » ou « sans atelier », fondé sur des exercices de

(1) C. Defodon, « Les travaux du congrès du Havre... », art. cit.; C. D. [Charles Delon], « La question du travail manuel devant la réunion des directeurs d'écoles de Paris », Manuel général de l'instruction primaire, 1889, partie générale, pp. 215-216.

(2) Gustave Philippon, Le Travail manuel à l'école primaire. Conférence faite à l'école normale de Laon, Paris, Larousse, 1888, p. 16.

(3) Exposition universelle internationale de 1900..., op. cit., p. 109.

(4) R. Leblanc, «L'enseignement professionnel à l'école élémentaire et à l'école normale », L'Enseignement manuel et expérimental, août 1889, pp. 194-198; id., « Les travaux manuels dans les écoles normales d'instituteurs », Revue pédagogique, $1^{\text {er }}$ semestre 1891, pp. 8-13. Comme le réclame Leblanc, l'arrêté du 3 janvier 1891 inscrit les « travaux en papier et en carton » au programme de première année des écoles normales d'instituteurs. Dans le même temps, la place des ouvriers est minorée: considérés comme des « auxiliaires du maître », ils ne doivent, « en aucune façon, s'immiscer dans l'enseignement lui-même» (Circulaire du 12 mars 1891, BAMIP, t. 49, pp. 280-282). 
tissage, pliage, découpage et cartonnage en partie inspirés du programme des écoles maternelles. «Les programmes officiels réservent ce travail sans atelier aux cours élémentaire et moyen, mais on peut également le répartir sur les trois années de l'école primaire, y compris le cours supérieur », affirme ainsi Philippon, qui reconnaît par ailleurs le caractère exceptionnel des exercices d'atelier (1). L'iconographie présentée dans le rapport de l'exposition universelle de 1900 relatif à l'enseignement primaire, qui réserve les photographies d'ateliers aux écoles primaires supérieures et aux cours du soir de la ville de Paris, est particulièrement significative de ce nouvel état d'esprit (2).

\section{L'association aux mathématiques et au dessin : un travail manuel « avant tout éducatif »}

Joël Lebeaume a mis en évidence le rôle essentiel de Leblanc, principal promoteur et théoricien de cette réévaluation (3). Ce dernier apparaît en effet comme un personnage central. Dès le milieu des années 1880, il développe activement ses conceptions dans différentes revues: L'Instruction primaire, le Manuel général, la Revue pédagogique, et surtout L'Enseignement manuel et expérimental, qu'il fonde en 1888 et dont un des premiers articles porte précisément sur le « choix des exercices de travail manuel dans les écoles primaires élémentaires dépourvues d'atelier » (4). En 1890, devenu inspecteur général en remplacement de Salicis, décédé, il participe à l'élaboration du nouveau programme de travail manuel des écoles parisiennes: il s'agit de rendre cet enseignement moins dispendieux, conformément aux souhaits de certains représentants de la municipalité, mais aussi d'en faire un élément à part entière de l'éducation générale. Pour Leblanc, celui-ci doit être « subordonné aux exigences et aux nécessités scolaires »(5), c'est-à-dire être dispensé à peu de

(1) G. Philippon, Le Travail manuel à l'école primaire..., op. cit., pp. 5 et 14.

(2) Exposition universelle internationale de 1900..., op. cit.

(3) J. Lebeaume, Cent ans de travail manuel..., op. cit., pp. 140-154, ainsi que les documents publiés en annexe, pp. 61-74.

(4) R. Leblanc, « Du choix des exercices de travail manuel dans les écoles primaires élémentaires dépourvues d'atelier ", L'Enseignement manuel et expérimental, août 1888, pp. 17-19.

(5) R. Leblanc, « Programme de travaux manuels proposé pour les écoles élémentaires de la ville de Paris », L'Enseignement manuel et expérimental, septembre 1890, p. 179. 
frais - de préférence dans la classe -, s'accorder aux capacités physiques des enfants, et surtout ne pas constituer une matière isolée de l'enseignement, qui porterait préjudice aux enseignements fondamentaux et serait du même coup mal acceptée par les maîtres. Aussi prône-t-il un enseignement manuel fortement lié à celui de la géométrie et du dessin, selon une formule qui va effectivement se développer dans la décennie 1890, non seulement dans la capitale mais aussi en province.

Cet enseignement manuel, Leblanc le veut « avant tout éducatif » : « Il faut donc non seulement que le travail manuel contribue à l'éducation physique, mais il faut encore qu'il prête son concours à l'éducation intellectuelle en apportant à la partie scientifique (dessin, formes géométriques, calcul) le concret qui lui fait si souvent défaut dans l'enseignement ordinaire »(1). Dans le programme parisien, chaque exercice manuel est associé à une notion mathématique et à une activité graphique, dessin ou croquis coté. Fournissant « les meilleurs moyens d'intuition », le travail manuel est ainsi envisagé comme la partie expérimentale, ou appliquée, de l'enseignement mathématique (et plus particulièrement de la géométrie qui, depuis 1882, commence dès l'entrée à l'école), au même titre que les manipulations ou les travaux agricoles dans l'enseignement des sciences physiques et naturelles. Certaines des vertus éducatives des sciences expérimentales lui sont d'ailleurs attribuées, telles que le développement de l'esprit d'observation et de la réflexion. On assiste donc là à un élargissement du champ des finalités de l'enseignement manuel qui, en favorisant l'observation et la manipulation d'objets sensibles, participe de la rénovation pédagogique entreprise par les républicains. Réclamé dès le début du $\mathrm{Xx}^{\mathrm{e}}$ siècle (2) son rattachement à l'éducation intellectuelle sera entériné avec le nouveau plan d'études des écoles primaires élémentaires de 1923.

(1) Ibid., p. 180. Cf. également « Cours temporaire de travail manuel pendant les vacances scolaires. Résumé des conférences faites à l'école normale de Carcassonne par M. R. Leblanc aux instituteurs qui ont suivi les cours de travail manuel organisés pendant les vacances de 1887 », Manuel général de l'instruction primaire, 1887, partie générale, pp. 690-692 et 703-704.

(2) Henri Gilbault, « Le travail manuel dans les écoles primaires de garçons », Revue pédagogique, $1^{\text {er }}$ semestre 1906, pp. 56-69. 
Théorisé par Leblanc et officialisé à Paris en 1891, le principe d'une connexion entre enseignement manuel, enseignement mathématique et enseignement graphique n'est cependant pas créé de toutes pièces. Il s'insère dans un mouvement plus large qui s'appuie sur des idées, parfois anciennes (1), qui circulent dans des revues pédagogiques ou dans certains manuels et nourrissent des pratiques de classes effectives. Conservé aux archives départementales de Seine-Maritime, un recueil des exercices de travaux manuels exécutés à l'école Louis Vauquelin de Rouen durant l'année scolaire 18861887 apporte un témoignage concret de l'existence de telles pratiques avant 1890. Il réunit une série de découpages et de pliages exécutés à partir des exercices proposés par la revue L'Instruction primaire au cours de cette même année scolaire. Ceux-ci sont précédés d'un petit texte de présentation du directeur de l'école, qui souligne le triple avantage - économique, éducatif et proprement disciplinaire - d'un tel enseignement: « $1^{\circ} \mathrm{Il}$ n'exige aucun matériel spécial et les cahiers finis, même les cahiers de brouillon, fournissent tout le papier nécessaire aux exercices; $2^{\circ}$ Il répond aux besoins des enfants qui aiment tant à s'amuser avec du papier, et qui trouvent là l'utile en même temps que l'agréable; $3^{\circ} \mathrm{Il}$ nous sert d'auxiliaire dans l'enseignement du dessin et de la géométrie » (2). Si elle n'est pas énoncée explicitement, la dimension collective de cet enseignement mérite également d'être signalée, puisque les élèves de tous les cours (élémentaire, moyen, supérieur) ont pu y prendre part.

\section{Une « vraie » discipline?}

Ainsi envisagé, le travail manuel «sans atelier » redessine assez largement les contours de la discipline. À l'externalisation - envisagée, réclamée ou véritablement effective - des premières années, succède la volonté affirmée de son incorporation. Moins exigeant en termes de locaux scolaires, il peut intégrer l'espace de la classe pour devenir, à l'instar des autres disciplines, l'objet d'un enseignement collectif plus conforme à la pédagogie officielle. Réunis dans la même classe, tous les élèves effectuent en même temps le même

(1) Voir par exemple les articles de Gustave Francolin dans la revue L'Enseignement professionnel, 15 novembre 1864, pp. 403-404, et 15 mars 1865, pp. 469-470.

(2) Archives départementales de la Seine-Maritime, T 2380. Ce recueil est publié sur la page web http://www.inrp.fr/she/illustrations_tm. Nous remercions Anne Bidois qui a bien voulu en réaliser les photographies. 
travail, ce qui est rarement possible avec les exercices d'atelier, faute de matériel suffisant. Comme dans les leçons d'écriture, le maître dessine le modèle au tableau noir, donne des explications orales à l'ensemble des élèves, en procédant au besoin par demandes et réponses comme le suggèrent certains manuels, puis circule dans la classe pour vérifier ou corriger les travaux de chacun. Dans les écoles mixtes, garçons et filles peuvent même recevoir une partie de l'enseignement en commun, la réalisation matérielle - pliages et découpages ou points de couture - marquant seule la différence entre les premiers et les secondes (1). La disqualification du bois et du fer au profit de matériaux peu onéreux et d'encombrement réduit (laine, papier, carton) renforce l'entrée de l'enseignement manuel dans une certaine normalité scolaire, grâce notamment à l'emploi d'un cahier spécial dans lequel sont conservés les travaux des élèves, accompagnés de croquis, dessins, calculs, vocabulaire, définitions ou explications écrites. Relevé par le maître à la fin de chaque leçon, ce cahier conserve la trace du (bon) déroulement de l'enseignement dispensé, tout comme le cahier de devoirs mensuels visé régulièrement par les parents (2). Enfin, alors que l'enseignement en atelier bute sur la question d'une organisation rationnelle des savoirs manuels (liée notamment à celle d'une classification raisonnée des gestes, des matériaux et des outils) commandant la gradation des apprentissages (3), le lien quasi-exclusif avec les mathématiques, et plus particulièrement avec la géométrie, discipline ordonnée s'il en est, et le dessin géométrique, permet d'envisager un agencement méthodique et véritablement gradué des exercices manuels, dont la rationalité apparaît dès lors incontestable. Question vitale aux yeux des acteurs, pour qui le rationnel et le progressif signalent la pertinence d'une méthode, et, au-delà, la légitimité de la discipline. Chez certains auteurs, cet

(1) Le Nouveau journal de la classe, 1906.

(2) M. Coste, J. Lapassade, Le Travail manuel à l'école primaire, Paris, A. Jeandé, Pau, Lafon /Vve Ribaut/ Tonnet, s.d. [1892], pp. 12-14; Alexis Jully, Émile Rocheron, Le Travail manuel à l'école primaire (classes sans ateliers), Paris, Belin, 1892, p. 15. Sur ce point, voir J. Lebaume, Cent ans de travail manuel..., op. cit., pp. 146-147.

(3) Cette question est examinée en 1865 par Gustave Francolin et Louis Guémied dans la revue L'Enseignement professionnel, pp. 430-431 et 445-447: le premier propose un «tableau des actions et des opérations avec lesquelles les élèves doivent être familiarisés », tandis que le second étudie la possibilité de « ramener [le travail manuel] à quelques opérations fondamentales ». Elle est reprise en 1872 par O. Gréard, dans son mémoire sur les écoles d'apprentis, où Guémied est largement cité. 
ancrage dans les mathématiques se prolonge jusque dans les exercices d'atelier, considérés non plus en référence au métier, mais comme la suite naturelle des travaux de pliage ou de découpage.

Cette conversion épistémologique n'est pas sans rappeler celle qui s'était opérée au tournant de la décennie 1880 lors de la grande réforme de l'enseignement du dessin, plaçant celui-ci sous l'égide de la géométrie et favorisant ainsi sa reconnaissance en tant que « vraie » discipline (1). L'association du travail manuel et du dessin est d'ailleurs revendiquée. Elle trouve sa consistance dans l'ancrage de ces deux enseignements dans la géométrie, mais aussi dans une communauté de finalités, au premier rang desquelles figure l'éducation de l'œil et de la main. Elle résulte aussi du fait que le programme de dessin arrêté en 1881, et c'est alors quelque chose d'une réelle nouveauté, accorde une large place aux représentations spatiales: les activités de pliage, de découpage ou de cartonnage permettent de réaliser concrètement les formes géométriques ou les motifs décoratifs étudiés dans le cadre du cours de dessin. Dans les années 1900, l'inspecteur du dessin Jules Pillet, fervent partisan d'une alliance entre les deux disciplines, soulignera tout l'intérêt de ces exercices qui « font suffisamment pénétrer l'élève dans le domaine des trois dimensions, pour qu'il puisse ensuite comprendre [...] la représentation géométrale ou cavalière d'un objet quelconque » (2). De même, les activités de modelage inscrites au programme de travail manuel des écoles élémentaires - solides géométriques au cours élémentaire, ornements de faible relief au cours moyen -, qui peuvent se prolonger dans des exercices de stéréotomie, sont fortement connectées à l'enseignement du dessin (3). Non seulement les modèles employés sont généralement les mêmes pour les deux types d'activité, mais chaque exercice de modelage est précédé ou suivi de l'exécution du dessin correspondant, favorisant les allers-retours entre le plan et l'espace.

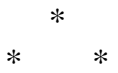

(1) Renaud d'Enfert, Daniel Lagoutte, Un Art pour tous. Le dessin à l'école de 1800 à nos jours, Lyon, INRP, 2004, pp. 36-45.

(2) Jules Pillet, L'Enseignement du dessin en France dans les établissements universitaires (1878-1904), Paris, Librairie des arts du dessin, s.d. [1904], p. 68.

(3) G. Salicis, Instruction spéciale..., op. cit., p. 27; Georges Dumont, Gustave Philippon, Guide pratique des travaux manuels, Paris, Larousse, 1888, pp. 100-102 et 
L'histoire de l'enseignement du travail manuel dans les écoles primaires élémentaires de garçons apparaît donc sensiblement différente de celle que nous livre généralement l'historiographie. Une focalisation excessive sur ce qui semblait la seule forme légitime de travail manuel, à savoir le travail en atelier du fer et du bois, a conduit à en négliger (ou à en déprécier) des modalités moins visibles, car plus scolaires. Porté en 1882 au programme du cours élémentaire et du cours moyen puis mis à l'honneur quelques années plus tard, notamment par Leblanc, le travail manuel «élémentaire », ou "sans atelier », ou « éducatif », est une composante essentielle de cet enseignement, qui pénètre, avec plus ou moins de succès, les pratiques enseignantes via son association avec ceux de géométrie et de dessin.

Cette association des trois disciplines - travail manuel, dessin, mathématiques - n'est pas officialisée dans l'immédiat, au niveau national du moins. Dans l'enseignement élémentaire, il faut attendre les instructions ministérielles de 1923 pour qu'elle soit explicitement reconnue, alors que c'est le cas dès 1909 pour l'enseignement primaire supérieur. Elle n'en devient pas moins opératoire à partir des années 1890: à Paris, on l'a vu, mais aussi en d'autres lieux; à l'instigation de la hiérarchie locale, ou par l'intermédiaire des manuels scolaires et des revues pédagogiques qui publient, tableaux de « concordance » à l'appui, des répartitions annuelles ou mensuelles des programmes mettant en parallèle notions géométriques, exercices graphiques et activités manuelles (1). Des travaux d'élèves portent témoignage d'une réelle mise en pratique de ces collaborations disciplinaires: vers 1900, dans la classe de Frédéric Boni, instituteur à Nice, dessin géométrique (à la règle et au compas), dessin à vue, travail manuel et coloriage, mais aussi calculs de surfaces, forment les différentes facettes d'une même leçon (2).

167-174. Un autre intérêt des exercices de stéréotomie est de pouvoir faire collaborer les élèves à un projet commun, par exemple la réalisation d'une voûte.

(1) P. Laurent, « Dessin et travaux manuels. Notre programme », L'Instruction primaire, 1889-1890, pp. 15-16 et 136; Palmyre Martin, L'Année préparatoire de travail manuel, Paris, A. Colin, 1893, p. 4 (publié par J. Lebaume dans École, technique et travail manuel, op. cit., p. 62); Jules Pouillot, « Instruction sur l'enseignement du dessin au cours moyen dans les écoles élémentaires », Revue pédagogique, $1^{\text {er }}$ semestre 1898, pp. 232-238, 337-351 et 442-451. Les conférences pédagogiques sont également un stimulant efficace. $C f$. "Conférence des instituteurs du Mans », L'Enseignement manuel et expérimental, juin 1891, pp. 130-132.

(2) Album conservé à la bibliothèque de l'INRP et contenant les dessins réalisés dans cette école par l'élève Ignace Brun, 12 ans, au cours de l'année scolaire 1900- 
On pourra objecter que cette association tripartite se fait au détriment de la discipline intitulée « travail manuel », que les exercices de pliage, de découpage ou de modelage ne sont pas du vrai travail et que le travail manuel ainsi conçu est relégué au rang de discipline de service. En réalité, qu'il s'agisse de travaux d'atelier ou d'exercices de découpage, les finalités assignées restent largement similaires: il s'agit d'exercer l'œil et la main et de donner le goût de l'activité manuelle. De plus, l'association des trois disciplines n'est pas sans portée heuristique: elle vise à inculquer aux élèves l'idée que le travail manuel - et plus généralement l'exercice du métier - ne relève pas de pratiques empiriques et mobilise au contraire, en tant qu'activité raisonnée, des connaissances mathématiques et des compétences graphiques. Par ailleurs, il faut resituer l'introduction du travail manuel dans le contexte plus général de la décennie 1880. Avec la loi du 28 mars 1882, non seulement le travail manuel, mais aussi les sciences, le dessin, la musique, la gymnastique, les exercices militaires viennent s'intercaler dans l'emploi du temps des élèves. Face à cette « surcharge » des programmes, sinon réelle, du moins vécue comme telle, le travail manuel aurait-il pu se constituer en discipline totalement autonome? Si le succès escompté n'est pas toujours au rendez-vous - Leblanc dresse en 1900 un bilan nuancé de son développement, déplorant notamment sa suppression de certains programmes départementaux (1) -, la formule de l'association à d'autres matières, confirmée par les instructions de 1923, a contribué de façon décisive, sur le long terme, à son inscription dans le cursus de l'école élémentaire - première étape avant les remises en question et les renouvellements qui caractérisent le devenir de bien des disciplines.

Il ne s'agit pas, pour autant, d'éluder la problématique de l'atelier scolaire. Au terme de cette étude, un certain nombre de questions subsistent, qui méritent d'être travaillées plus avant. Plutôt que de raisonner en termes de succès ou d'échec, il convient de s'interroger sur la place effective de l'atelier dans le parcours scolaire des élèves du primaire et de considérer son articulation avec d'autres formes, non moins légitimes, d'enseignement manuel. Plus, peut-être, que les autres disciplines de l'école primaire, le travail manuel exige pour

1901. Voir aussi J. Lebeaume, École, technique et travail manuel, op. cit., p. 68, qui présente le cahier de devoirs mensuels d'un élève de l'école de garçons d'Ollé (Eureet-Loir).

(1) Exposition universelle internationale de 1900..., op. cit., pp. 991-992. 
cela qu'une attention particulière soit accordée aux réalités locales. L'échelle du département semble particulièrement pertinente pour identifier les spécificités géographiques, démographiques, économiques, dont dépendent des politiques scolaires localement différenciées, et pour saisir le jeu des acteurs - inspecteurs d'académie, municipalités, conseils généraux - qui soutiennent ou au contraire combattent l'installation d'un atelier, mais peut-être aussi s'en désintéressent tout simplement. C'est également à ce niveau qu'une cartographie des ateliers scolaires peut être envisagée, en exploitant les fonds d'archives départementaux et municipaux (1). Celle-ci doit combiner approche quantitative et approche qualitative. Car si on ne peut se satisfaire de quelques exemples glanés au gré des recherches, il ne suffit pas non plus de dénombrer les ateliers créés dans les écoles élémentaires à la fin du XIX ${ }^{\mathrm{e}}$ siècle. Il est nécessaire d'en préciser les caractéristiques, comme l'environnement et le contexte scolaire, l'organisation matérielle et les ressources financières, l'enseignement dispensé et le personnel mobilisé, l'âge, le niveau de scolarisation et le nombre des élèves concernés. En ayant à l'esprit que des formes inattendues d'enseignement manuel peuvent se développer à l'extérieur de l'école, parfois sous son contrôle. Et que la rareté, vraisemblable, des ateliers scolaires n'interdit pas de rechercher les traces, matérielles notamment, d'autres modalités d'enseignement manuel.

Renaud d'ENFERT

IUFM de l'académie de Versailles

(1) C'est également l'échelle du département qu'a adoptée Gérard Bodé pour la constitution, actuellement en cours, d'un répertoire des établissements d'enseignement technique. Voir Gérard Bodé (dir.), Les Établissements d'enseignement technique en France, 1789-1940. Tome 1: La Corrèze; Tome 2: Le Lot; Tome 3: Les Côtesd'Armor, Lyon, INRP, 2004-2006. 